\title{
Colloidal Stability and Re-dispersibility of Mesoporous Silica Nanoparticles in Biological Media
}

Andressa da Cruz Schneida ${ }^{a}$, Camila Pedroso Silveira ${ }^{a}$, Flávia Elisa Galdino a,b, Larissa Fernanda Ferreira ${ }^{\mathrm{a}, \mathrm{c}}$, Karim Bouchmella ${ }^{\mathrm{d}}$ and Mateus Borba Cardoso $\mathrm{a}^{\mathrm{a}, \mathrm{b}, \mathrm{c} *}$

a. Laboratório Nacional de Luz Sincrotron (LNLS) and Laboratório Nacional de Nanotecnologia (LNNano), Centro Nacional de Pesquisa em Energia e Materiais (CNPEM), CEP 13083-970, Campinas, São Paulo, Brasil.

b. Instituto de Química (IQ), Universidade Estadual de Campinas (UNICAMP), CEP 13083-970, Caixa Postal 6154, Campinas, SP, Brasil.

c. Pós Graduação em Biotecnociência, Universidade Federal do ABC, CEP 09210-580, Santo André, Brasil.

d. Institut Charles Gerhardt Montpellier, UMR-5253 Univ Montpellier, CNRS, ENSCM, cc 1701, Place Eugène Bataillon, 34095 Montpellier Cedex 05, France.

*Corresponding author, email: cardosomb@Inls.br. 


\section{Mesoporous Silica Nanoparticles characterization}

1.1. CTAB removal from MSNs: Thermogravimetric Analysis (TGA)
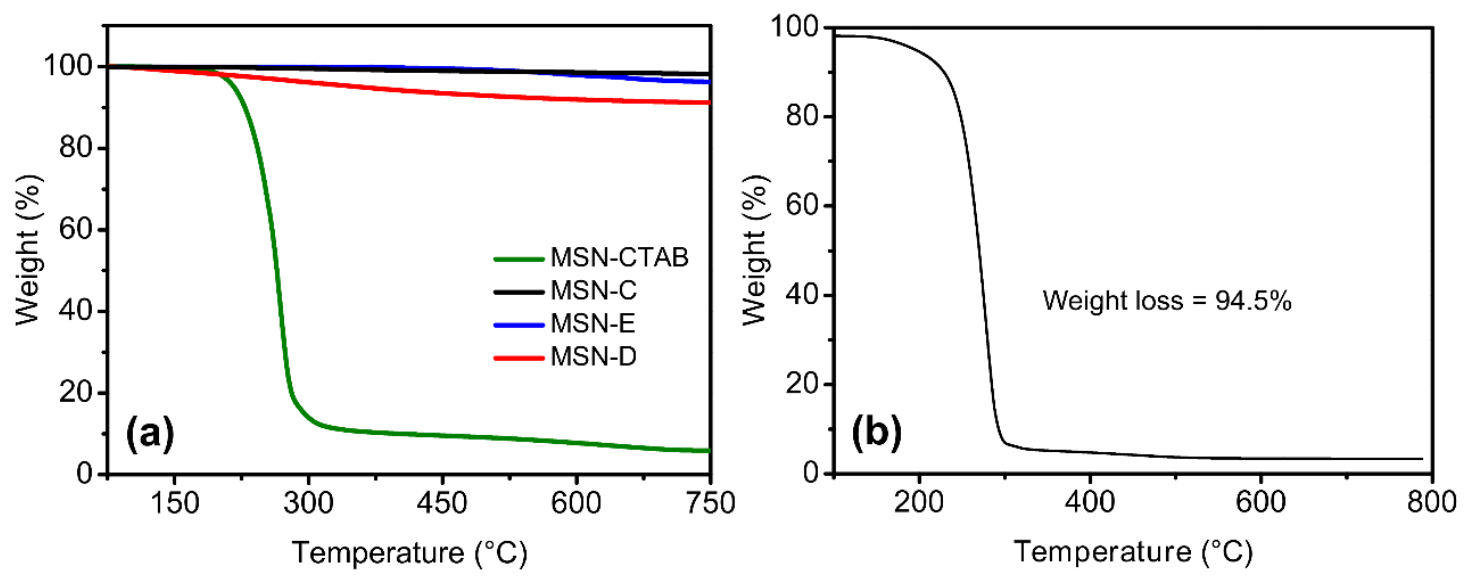

Figure S1. (a) Evaluation of CTAB removal efficiency by thermogravimetric analyses (TGA) of MSN-CTAB, MSN-C, MSN-E and MSN-D; (b) Non-normalized TGA curve for MSN-CTAB.

1.2. Influence of CTAB removal method on MSNs' structure: $\mathrm{N}_{2}$ adsorption/desorption isotherms and Scanning Electron Microscopy (SEM)

\subsubsection{Surface area and porosity}

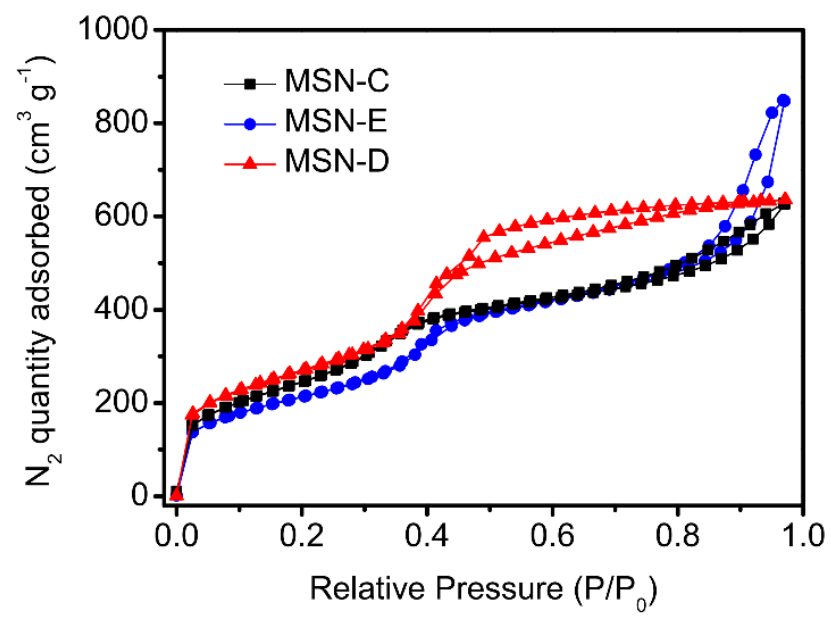

Figure S2. $\mathrm{N}_{2}$ Adsorption-desorption isotherms of MSN-C, MSN-E and MSN-D. 


\subsubsection{Morphology}

The effect of calcination, solvent extraction and dialysis on MSNs morphology was evaluated by SEM (Figure S3). The size distribution of MSNs (Figure S4) was determined using MSN-D images, since all the samples were obtained from the same original dispersion.
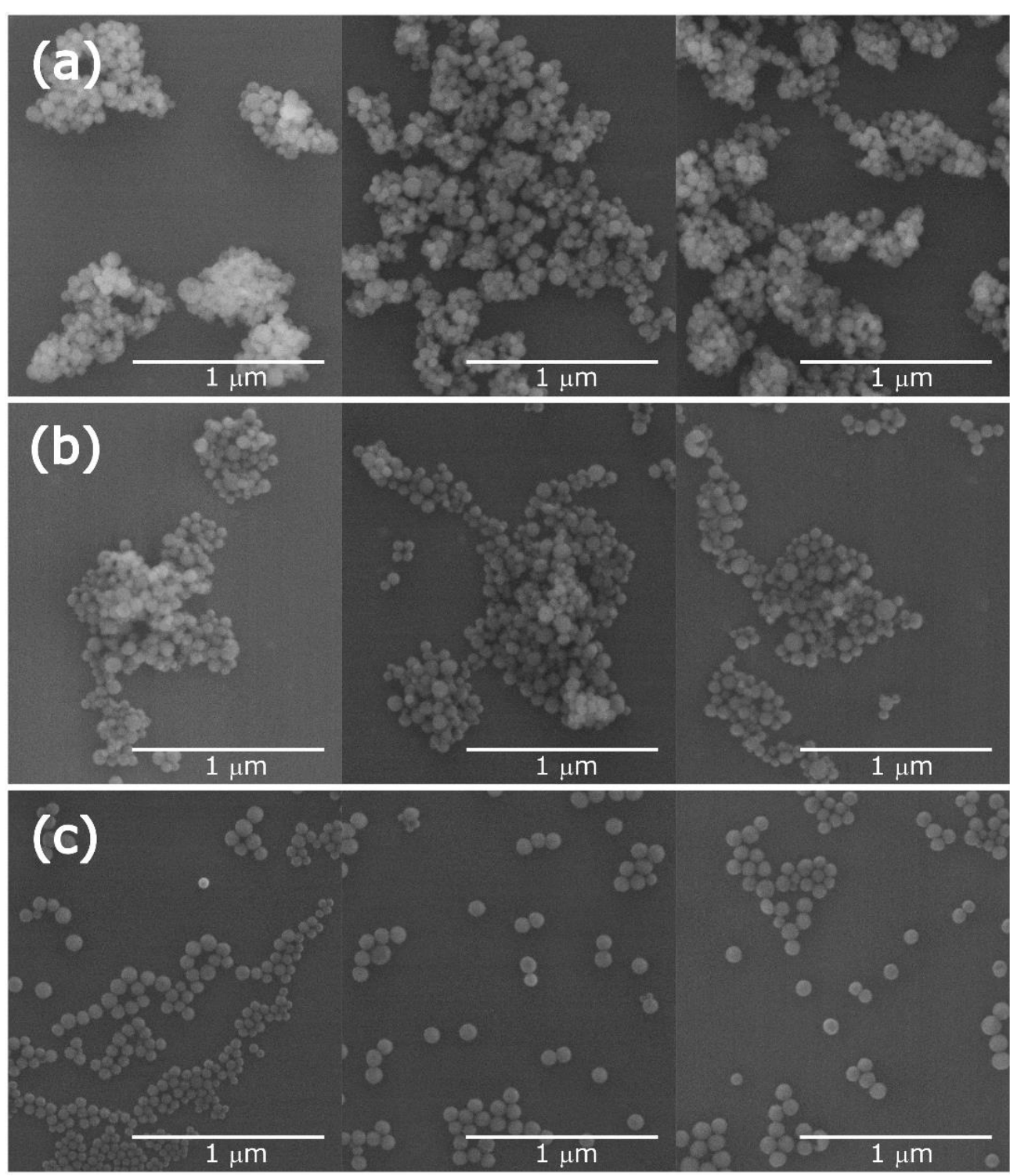

Figure S3. SEM image of (a) MSN-C, (b) MSN-E and (c) MSN-D. 

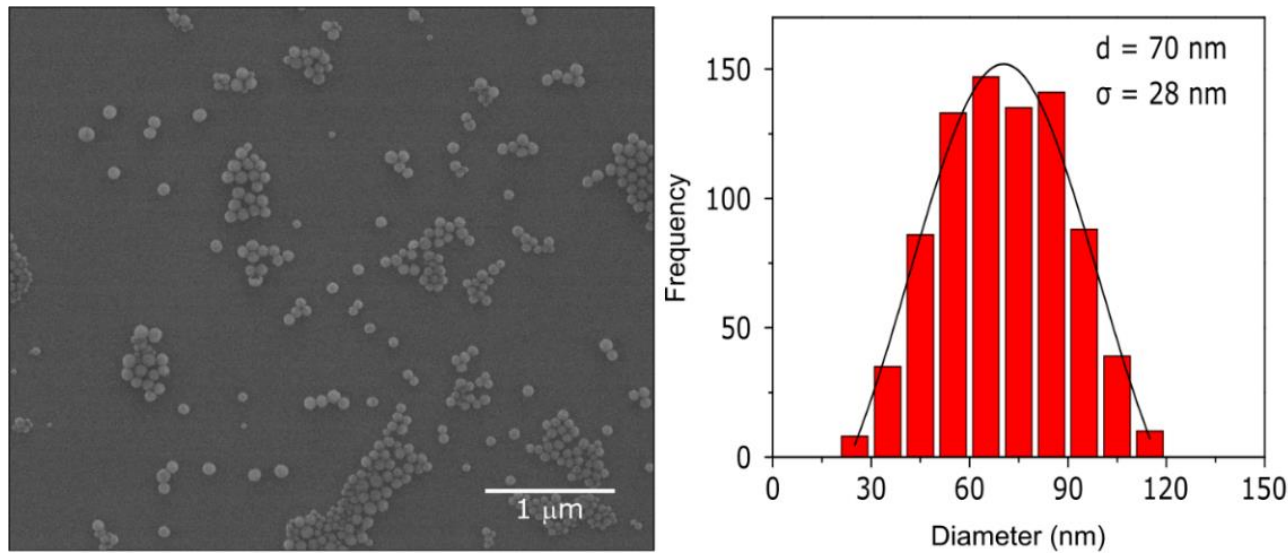

Figure S4. SEM image (left) and size distribution (right) of MSN-D, obtained by counting at least 800 particles.

\subsection{Colloidal stability of MSNs in water: Dynamic Light Scattering (DLS) and Differential Centrifugal Sedimentation (DCS)}

The DLS size distributions for MSN-C and MSN-E was not possible to extract because their autocorrelation functions were not typical exponentials (Figure S5). The correlation function $g_{(\tau)}$ can be written as:

$$
g_{(\tau)}=1+\beta e^{-2 D_{\tau} q_{2} \tau} \quad \text { Equation } 1
$$

where $\tau$ is the time increment; $\beta$ is the coherence factor, which is dependent on the detector area, optical alignment and scattering properties of the particles; $D$ is the diffusion coefficient and $q$ is the Bragg wave vector, which is proportional to the refractive index of the medium and inversely proportional to the wavelength of the incident beam light. By measuring the time correlation $\left(g_{(\tau)}\right.$ over $\tau$ ), it is possible to get $D$, which is associated to the particle radius. ${ }^{1}$ From Equation 1 , the bigger the correlation function over time, the smaller the diffusion coefficient. Therefore, the bigger the particles the longer is the lag time for the autocorrelation to go to zero. From Figure S5, the increase in lag time for MSN-C and MSN-E are associated to bigger particles and the inflections observed in these samples to the presence of multiple populations. 


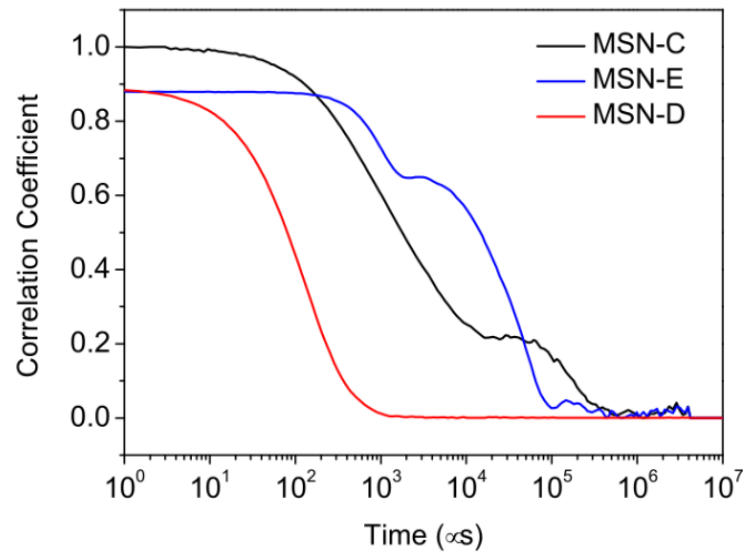

Figure S5. Correlations curves of MSN-C, MSN-E and MSN-D obtained by DLS.

To obtain trustable size distributions, these samples were evaluated using Differential Centrifugal Sedimentation (DCS). DCS consists of an analytical disk centrifuge that measures the sedimentation time of a sample of known density under centrifugal force through a gradient from the injection point to the detector. The sedimentation time varies with particle size, so bigger particles of a particular density take a shorter time to reach the detector than smaller particles of the same density (Figure S6). Therefore, based on Stokes-Einstein equations, ${ }^{2}$ DCS gives a size distribution assuming a uniform density throughout the particle. The results are presented in terms of "apparent diameter" because the real density of each set of MSNs (MSN-C and MSN-D) are not known and were assumed to be in the range of Stöber silica nanoparticles $\left(2.0 \mathrm{~g} \mathrm{~cm}^{-3}\right)$. Hence, the DCS results shown in Figure $3 \mathrm{~b}$ are shifted to smaller sizes in relation to the real values.

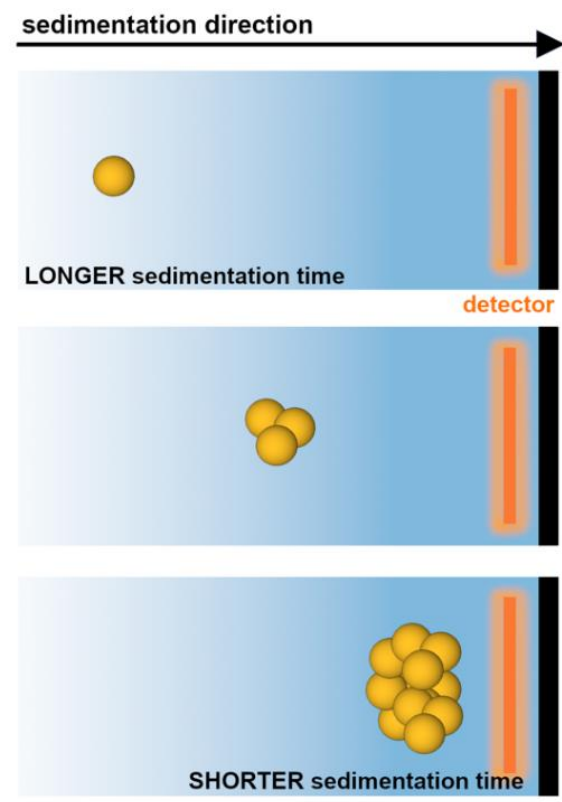

Figure S6. Schematic illustration of DCS running individualized particles (top), moderately aggregated particles (medium) and heavily aggregated particles (bottom). 


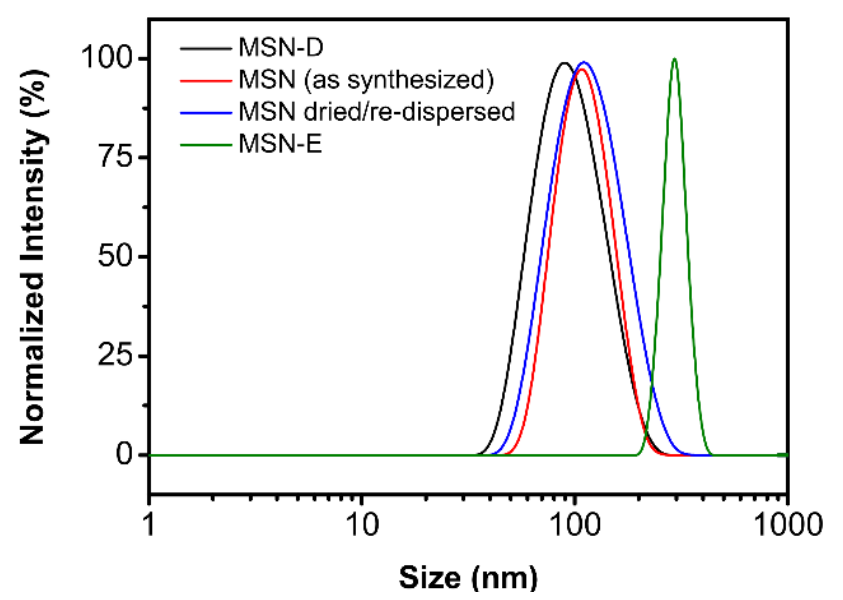

Figure S7. Size distribution obtained by DLS for MSN as synthesized, MSN dried and redispersed in water, MSN-D and MSN-E.
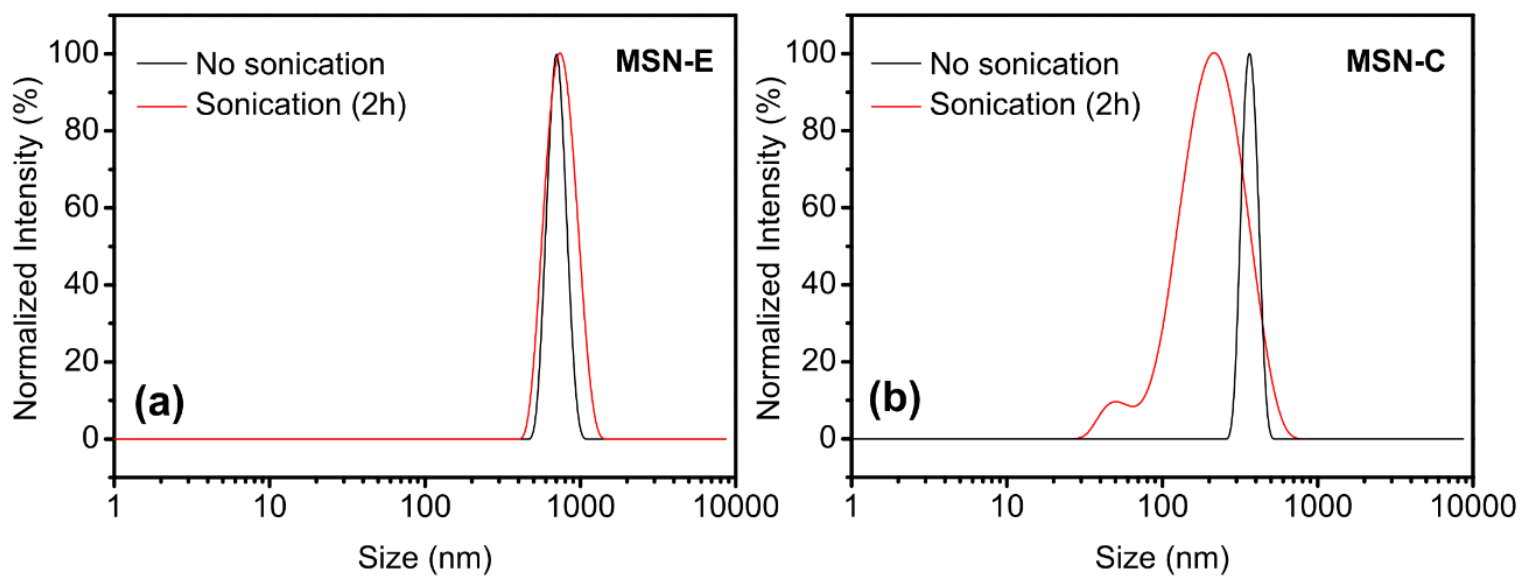

Figure S8. Size distribution (in water) obtained by DLS for (a) MSN-E and (b) MSN-C before and after $2 \mathrm{~h}$ of sonication. 
1.4. BSA-coated MSN: Thermogravimetric Analysis (TGA) and Dynamic Light Scattering $(D L S)$

Table S1. Hydrodynamic diameter in water of BSA-FD with different BSA:MSN ratios.

\begin{tabular}{cc}
\hline BSA:MSN ratio & Hydrodynamic diameter $(\mathrm{nm})$ \\
\hline $0: 1$ & 122 \\
\hline $1: 1$ & 124 \\
\hline $2: 1$ & 120 \\
\hline $5: 1$ & 121 \\
\hline $10: 1$ & 126 \\
\hline $20: 1$ & 127 \\
\hline $50: 1$ & 128 \\
\hline $100: 1$ & 130 \\
\hline
\end{tabular}

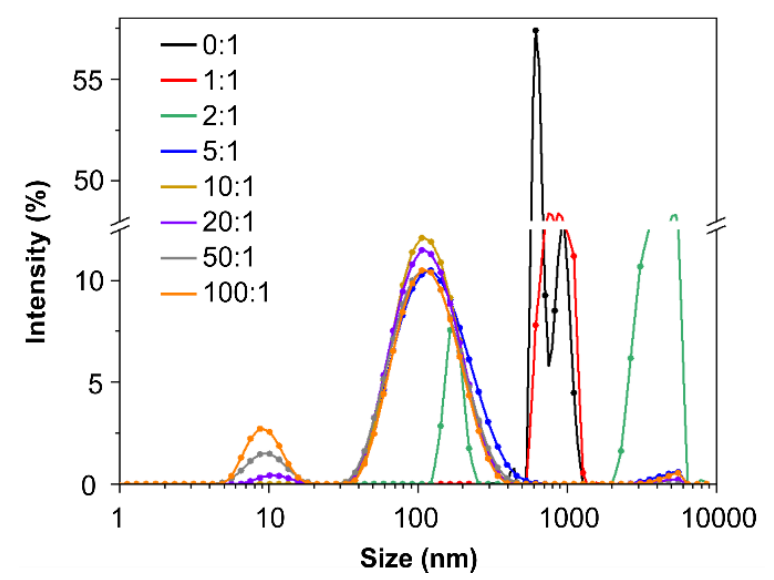

Figure S9. Evaluation of BSA:MSN ratio (in mass) influence on particles' colloidal stability in serum-free DMEM.

Complementary, DLS was applied to determine the hydrodynamic diameter variation of MSN-D after BSA coating (MSN-BSA) and freeze-drying (BSA-FD) (Figure S10). The measurements were performed in water using nanoparticle concentration of $0.5 \mathrm{mg} \mathrm{mL}^{-1}$. 


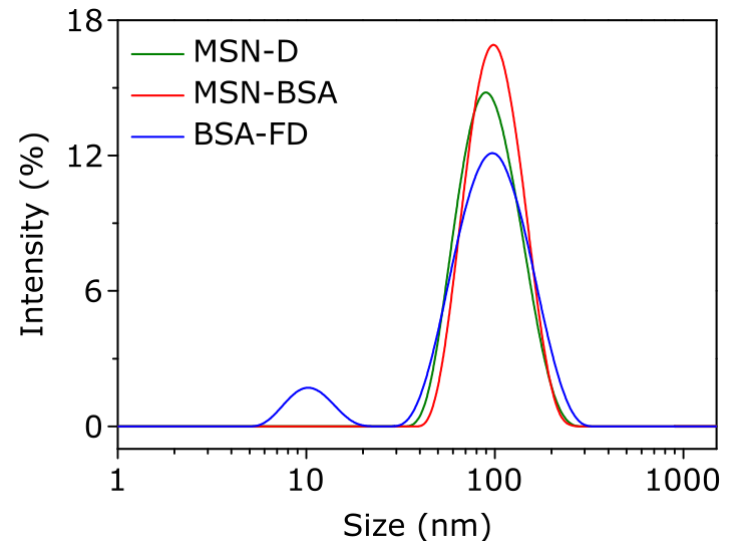

Figure S10. Size distributions by DLS in water of MSN-D (green), MSN-BSA (red) and BSA-FD (blue).

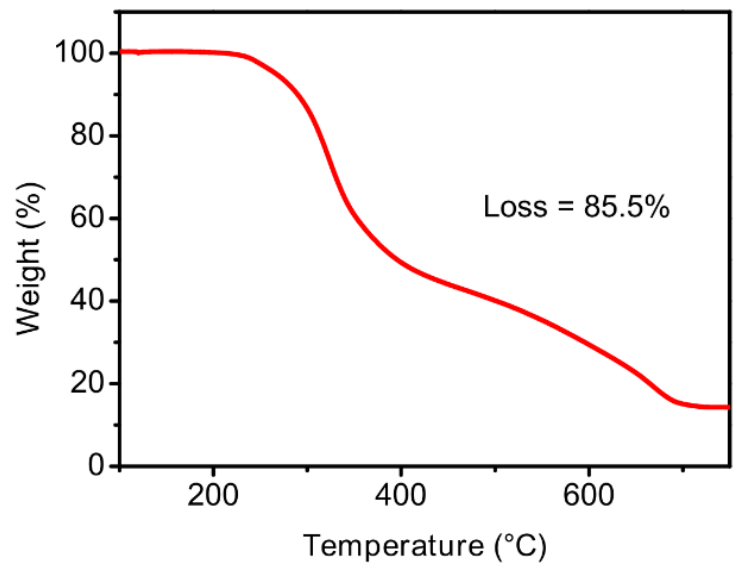

Figure S11. Evaluation of BSA content on BSA-FD sample by TGA.
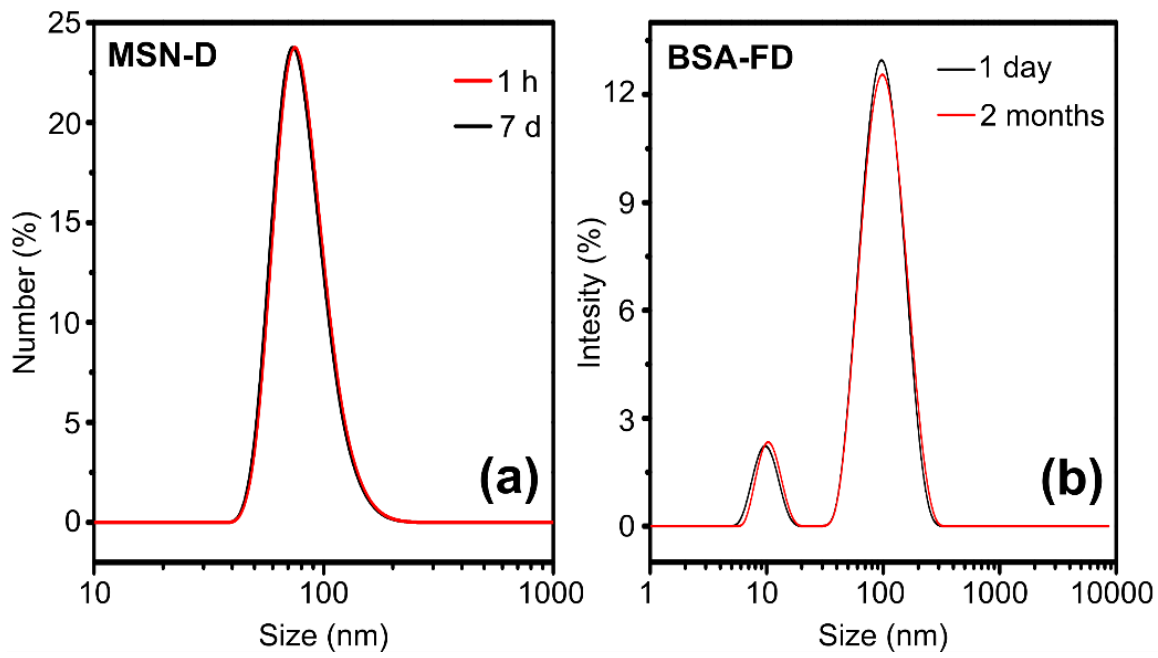

Figure S12. Colloidal stability over time for (a) MSN-D in ethanol (storage solvent),) and (b) BSAFD re-dispersed in DMEM (10\% FBS) after kept stored as a powder. 


\section{References}

1. Berne, B. J.; Pecora, R. Dynamic Light Scattering. Dover Publications, 2000.

2. Uchiyama, S.; Arisaka, F.; Stafford, W. F.; Laue, T. Analytical Ultracentrifugation. Springer, 2005. 